\title{
Flavanol monomer-induced changes to the human faecal microflora
}

\author{
Xenofon Tzounis ${ }^{1}$, Jelena Vulevic ${ }^{2}$, Gunter G. C. Kuhnle ${ }^{3}$, Trevor George ${ }^{1}$, Jadwiga Leonczak ${ }^{4}$, \\ Glenn R. Gibson ${ }^{2}$, Catherine Kwik-Uribe ${ }^{4}$ and Jeremy P. E. Spencer ${ }^{1}$ * \\ ${ }^{1}$ Molecular Nutrition Group, School of Chemistry, Food and Pharmacy, University of Reading, Reading RG2 6AP, UK \\ ${ }^{2}$ Food and Microbial Sciences Unit, School of Chemistry, Food and Pharmacy, University of Reading, Reading, RG2 6AP, UK \\ ${ }^{3}$ MRC Dunn Human Nutrition Unit, MRC/Wellcome Trust Building, Cambridge CB2 2XY, UK \\ ${ }^{4}$ Analytical and Applied Sciences Group, Mars, Incorporated, 800 High Street, Hackettstown, NJ 07840, USA
}

(Received 2 July 2007 - Revised 29 August 2007 - Accepted 25 September 2007)

We have investigated the bacterial-dependent metabolism of (-)-epicatechin and (+)-catechin using a pH-controlled, stirred, batch-culture fermentation system reflective of the distal region of the human large intestine. Incubation of $(-)$-epicatechin or $(+)$-catechin $(150 \mathrm{mg} / \mathrm{l} \mathrm{or}$ $1000 \mathrm{mg} / \mathrm{l})$ with faecal bacteria, led to the generation of 5-(3',4'-dihydroxyphenyl)- $\gamma$-valerolactone, 5-phenyl- $\gamma$-valerolactone and phenylpropionic acid. However, the formation of these metabolites from $(+)$-catechin required its initial conversion to $(+)$-epicatechin. The metabolism of both flavanols occurred in the presence of favourable carbon sources, notably sucrose and the prebiotic fructo-oligosaccharides, indicating that bacterial utilisation of flavanols also occurs when preferential energy sources are available. (+)-Catechin incubation affected the growth of select microflora, resulting in a statistically significant increase in the growth of the Clostridium coccoides-Eubacterium rectale group, Bifidobacterium spp. and Escherichia coli, as well as a significant inhibitory effect on the growth of the C. histolyticum group. In contrast, the effect of (- )-epicatechin was less profound, only significantly increasing the growth of the $C$. coccoides-Eubacterium rectale group. These potential prebiotic effects for both (+)-catechin and (-)-epicatechin were most notable at the lower concentration of $150 \mathrm{mg} / \mathrm{l}$. As both (-)-epicatechin and (+)-catechin were converted to the same metabolites, the more dramatic change in the growth of distinct microfloral populations produced by $(+)$-catechin incubation may be linked to the bacterial conversion of (+)-catechin to (+)-epicatechin. Together these data suggest that the consumption of flavanol-rich foods may support gut health through their ability to exert prebiotic actions.

Flavanols: Prebiotics: Faecal microflora: Large intestine

Representing one of the most important lifestyle factors, diet can strongly influence the incidence and onset of $\mathrm{CVD}^{(1)}$, and thus a healthy diet is an essential factor for healthy ageing $^{(2)}$. A number of dietary intervention studies in human subjects and animals, in particular those using Vitis vinifera (grape), Camellia sinensis (tea) and Theobroma cacao (cocoa) have demonstrated beneficial effects on vascular function $^{(3-5)}$. While such foods and beverages differ greatly in chemical composition and macro- and micronutrient content, they have in common that they are amongst the major dietary sources of flavanols. The in vivo effects of flavanols will be dependent on the absorption and metabolism of flavanols in the gastrointestinal tract. Studies have indicated that flavanols are subject to extensive metabolism by phase I and II enzymes to yield $O$-methylated, sulfated and glucuronidated forms during transfer from the small-intestinal lumen to the portal blood $^{(6)}$. However, significant amounts of ingested (-)-epicatechin, (+)-catechin, and their structurally related oligomeric forms (procyanidins), escape absorption in the small intestine, instead reaching the large intestine, where they encounter the resident colonic microflora ${ }^{(7)}$.
The human large intestine is an extremely active fermentation site and is inhabited by over 400 different bacterial species which number about $10^{14}$ colony-forming units in total ${ }^{(8)}$. The balance among these bacterial species has been linked to both beneficial and detrimental effects in the large intestine. For example, the progression of colorectal cancer and inflammatory bowel disease is associated with deleterious bacterial species, such as certain members of the Clostridium group, whilst others, such as Bifidobacterium and Lactobacillus spp., are known to exert beneficial effects in the colon ${ }^{(9-12)}$ and have been utilised in the development of probiotic functional foods ${ }^{(8,13)}$. In addition, there has been much recent interest in the development of 'prebiotics', defined as non-digestible food ingredients that have a beneficial effect on the host by selectively stimulating the growth of a limited number of specific bacterial strains in the large intestine ${ }^{(14)}$. For a nutrient to be classified as a prebiotic it has to fulfil three criteria: (a) it must undergo a low level of hydrolysis or absorption in the stomach and/or small intestine; (b) it must be selective for beneficial commensal bacteria in the colon by encouraging the growth and metabolism of these organisms; (c) it must alter the microflora to a healthy composition by inducing beneficial

Abbreviations: FISH, fluorescent in situ hybridisation; FOS, fructo-oligosaccharides; FRAP, ferric-reducing antioxidant power.

* Corresponding author: Dr Jeremy P. E. Spencer, fax +44 118931 0080, email j.p.e.spencer@reading.ac.uk 
luminal or systemic effects within the host ${ }^{(15)}$. Presently, the most widely used prebiotics are fructo-oligosaccharides (FOS) and galacto-oligosaccharides and studies have indicated the bifidogenic and anti-cancer potential of these carbohydrates ${ }^{(16-18)}$. However, whilst soyabean isoflavones have been shown to possess prebiotic potential in vivo ${ }^{(19)}$, there is limited information regarding the potential prebiotic potential of other classes of flavonoids, including the flavanols.

Previous in vitro investigations have indicated that flavanols, including procyanidins, are catabolised by the colonic microflora to low-molecular-weight compounds such as phenylacetic, phenylpropionic and phenylvaleric acids, which are mono-hydroxylated primarily in the meta or para position ${ }^{(20)}$. Furthermore, (-)-epigallocatechin and (-)-epicatechin have been shown to be metabolised to 5-( $3^{\prime}, 4^{\prime}, 5^{\prime}$-trihydroxyphenyl)$\gamma$-valerolactone and 5-(3',4'-dihydroxyphenyl)- $\gamma$-valerolactone by the intestinal microflora ${ }^{(21)}$. A recent in vitro study indicated that the fermentation of the tea polyphenols, catechin, epicatechin, $3^{\prime}-O$-methyl gallic acid, gallic acid and caffeic acid, by various pure isolates of pathogenic and commensal intestinal bacterial species leads to the generation of a variety of aromatic metabolites. Incubation of these polyphenols with the pure bacterial isolates significantly inhibited the growth of certain pathogenic bacterial species compared with other commensal anaerobes ${ }^{(22)}$. The aim of the present study was to investigate the potential of the flavanol monomers, $(-)$-epicatechin and $(+)$-catechin, to influence the growth of specific bacterial groups in a $\mathrm{pH}$-controlled, stirred, batch-culture fermentation system that is reflective of the environmental conditions located in the distal region of the human large intestine. We detail the differential metabolism of flavanols in this competitive bacterial environment and show that they are capable of inducing positive changes in the balance of bacterial groups.

\section{Materials and methods}

\section{Materials}

Unless otherwise stated, all chemicals and reagents were obtained from Sigma-Aldrich Co. Ltd (Poole, Dorset, UK) or Fisher (Loughborough, Leics, UK). Bacteriological growth media supplements were obtained from Oxoid Ltd (Basingstoke, Hants, UK). (+)-Catechin ( $>99 \%$ pure) and ( - )-epicatechin $(>90 \%$ pure) were purchased from Sigma-Aldrich. $(+)$-Epicatechin was obtained from Mars, Inc. (Hackettstown, NJ, USA). Raftilose P95 FOS was purchased from Orafti (Tienen, Belgium). HPLC columns were purchased from Waters Co. (Watford, Herts, UK) and Sumika Chemical Analysis Service (Singapore). Isopore $(0 \cdot 2 \mu \mathrm{m})$ membrane filters were obtained from Millipore Corp. (Watford, Herts, UK). All the oligonucleotide probes used for fluorescent in situ hybridisation (FISH) were commercially synthesised and labelled with the fluorescent dye $\mathrm{Cy} 3$ by MWG-Biotech Ltd (Milton Keynes, Bucks, UK). Sterilisation of media and instruments was achieved by autoclaving at $121^{\circ} \mathrm{C}$ for $15 \mathrm{~min}$.

\section{Faecal sample preparation}

Faecal samples were collected from three separate individuals. All volunteers were in good health and had not ingested antibiotics for at least 6 months before the study. Samples were collected, on site, on the day of the experiment and were used immediately. The samples were diluted 1:10 (w/v) with anaerobic phosphate buffer $(0.1 \mathrm{M} ; \mathrm{pH} 7.4)$ and homogenised in a stomacher for $2 \mathrm{~min}$. Resulting faecal slurries from each individual (i.e. faecal samples were not pooled) were used to inoculate the batch-culture vessels.

\section{Batch-culture fermentation}

Batch-culture fermentation vessels ( $300 \mathrm{ml}$ volume; one vessel per treatment group) were sterilised and filled with $135 \mathrm{ml}$ basal nutrient medium (peptone water $(2 \mathrm{~g} / \mathrm{l})$, yeast extract $(2 \mathrm{~g} / \mathrm{l}), \mathrm{NaCl}(0.1 \mathrm{~g} / \mathrm{l}), \mathrm{K}_{2} \mathrm{HPO}_{4}(0.04 \mathrm{~g} / \mathrm{l}), \mathrm{KH}_{2} \mathrm{PO}_{4}(0.04 \mathrm{~g} / \mathrm{l})$, $\mathrm{NaHCO}_{3} \quad(2 \mathrm{~g} / \mathrm{l}), \quad \mathrm{MgSO}_{4} \cdot 7 \mathrm{H}_{2} \mathrm{O} \quad(0.01 \mathrm{~g} / \mathrm{l}), \quad \mathrm{CaCl}_{2} \cdot 6 \mathrm{H}_{2} \mathrm{O}$ $(0.01 \mathrm{~g} / \mathrm{l})$, Tween $80(2 \mathrm{ml} / \mathrm{l})$, haemin $(50 \mathrm{mg} / \mathrm{l})$, vitamin $\mathrm{K}_{1}$ (10 $\mu \mathrm{l} / \mathrm{l})$, L-cysteine $(0.5 \mathrm{~g} / \mathrm{l})$, bile salts $(0.5 \mathrm{~g} / \mathrm{l})$, resazurin $(1 \mathrm{mg} / \mathrm{l})$ and distilled water). The $\mathrm{pH}$ of the basal medium was adjusted to 7.0 and autoclaved before dispensing it to the vessels. Medium was then gassed overnight with $\mathrm{O}_{2}$-free $\mathrm{N}_{2}$. Before the addition of faecal slurry samples, the temperature of the basal nutrient medium was set to $37^{\circ} \mathrm{C}$ by using a circulating water-bath and the $\mathrm{pH}$ was maintained at $6 \cdot 8$, using an Electrolab $\mathrm{pH}$ controller, in order to mimic conditions located in the distal region of the human large intestine (anaerobic; $37^{\circ} \mathrm{C} ; \mathrm{pH}$ about $6 \cdot 8$ ). Vessels were inoculated with $15 \mathrm{ml}$ faecal slurry $(1: 10, \mathrm{w} / \mathrm{v})$ and batch cultures were run under anaerobic conditions for a period of $48 \mathrm{~h}$ during which samples $(3 \mathrm{ml})$ were collected at seven time points $(0$, $4,8,10,17,24$ and $48 \mathrm{~h}$ ) for FISH, flavanol metabolism analysis by HPLC and assessment of the total antioxidant capacity (ferric-reducing antioxidant power (FRAP) assay). Before FISH analysis, duplicate samples were fixed overnight at $4^{\circ} \mathrm{C}$ with $4 \%$ (w/v) paraformaldehyde. The remaining sample was centrifuged for $5 \mathrm{~min}$ at $1500 \mathrm{~g}$ and the supernatant fraction was removed and stored in sterile Eppendorf tubes $(1.5 \mathrm{ml})$ at $-20^{\circ} \mathrm{C}$ to be analysed later by HPLC and in the FRAP assay.

\section{Inoculation of substrate in the batch culture}

$(+)$-Catechin and (-)-epicatechin $(150 \mathrm{mg} / \mathrm{l}$ and $1000 \mathrm{mg} / \mathrm{l})$ were inoculated in stirring batch-culture vessels (one per treatment) containing faecal slurry $(1: 10, \mathrm{w} / \mathrm{v})$. These amounts were estimated to reflect lower and upper levels of flavanol monomer intake. We also investigated the metabolism of compounds $(1000 \mathrm{mg} / \mathrm{l})$ in the presence of sucrose $(1 \%, \mathrm{w} / \mathrm{v})$ and in the presence of a known prebiotic compound, FOS $(1 \%$, w/v). Control experiments incubating flavanol monomers in medium without faecal slurry inoculation were also run under anaerobic conditions.

\section{Bacterial enumeration}

In order to access the differences in bacterial population, FISH was used with oligonucleotide probes designed to target specific diagnostic regions of $16 \mathrm{~S}$ rRNA. The probes were commercially synthesised and labelled with the fluorescent dye $\mathrm{Cy} 3$. The bacterial groups studied for enumeration were Bifidobacterium spp., Bacteroides spp., Lactobacillus/Enterococcus spp., Clostridium coccoides-Eubacterium rectale group, C. histolyticum group and Escherichia coli using the 
specific probes Bif164, Bac303, Lab158, Erec482, His150 and Ec1531 respectively. For total bacterial count 4,6-diamidino2-phenylindole nucleic acid stain was utilised. Samples fixed in $4 \%$ paraformaldehyde overnight at $4^{\circ} \mathrm{C}$ were then centrifuged at $1500 \mathrm{~g}$ for $5 \mathrm{~min}$, washed twice with PBS (0.1 M; $\mathrm{pH} \mathrm{7.0)}$, re-suspended in a mixture of PBS-99\% ethanol $(1: 1, \mathrm{v} / \mathrm{v})$ and stored at $-20^{\circ} \mathrm{C}$ for at least $1 \mathrm{~h}$. This process was used for all samples except those where the Lab158 probe was used. Samples probed with Lab 158 were subjected to an additional enzyme step (25 mM-tri(hydroxymethyl)-aminomethane-HCl, $10 \mathrm{~mm}$-EDTA, $585 \mathrm{~mm}$-sucrose, $5 \mathrm{~mm}-\mathrm{CaCl}_{2}$, sodium taurocholate $(0.3 \mathrm{mg} / \mathrm{ml})$, lysozyme $(2 \mathrm{mg} / \mathrm{ml})$, lipase $(0 \cdot 1 \mathrm{mg} / \mathrm{ml}))$ for $60 \mathrm{~min}$ at $37^{\circ} \mathrm{C}$ in order to increase cell permeability.

Cell suspensions were then added to the filtered sterilised hybridisation mixture (30 mM-tri(hydroxymethyl)-aminomethane- $\mathrm{HCl}, 1.36 \mathrm{M}-\mathrm{NaCl}, 0.15 \%$ SDS, $\mathrm{pH} 7.2$ ) and were left overnight to hybridise at the appropriate temperature for each probe. Hybridised mixtures were vacuum filtered using $0.2(\mathrm{~m}$ pore-size isopore membrane filters (Millipore Corporation, Watford, Herts, UK) and filters were placed onto labelled glass slides. A drop of Slowfade (Molecular Probes, Eugene, OR, USA) was added to each and a cover slip was placed on top of each filter and slides were stored in the dark at $4^{\circ} \mathrm{C}$. Slides were examined after $60 \mathrm{~min}$ using a fluorescent microscope (Nikon Eclipse E400). 4,6-Diamidino-2-phenylindole-stained cells were examined under UV light and a DM510 light filter was used to count micro-organisms hybridised with the probes. For each slide, fifteen different fields of view were counted. In order to determine the changes in the bacterial population we used an 'index of specific bacteria' (ISB). The ISB was calculated using the following equation: $\mathrm{ISB}=(\mathrm{Ns}(\mathrm{T} 1)-\mathrm{Ns}(\mathrm{T} 0))-(\mathrm{Nc}(\mathrm{T} 1)-\mathrm{Nc}(\mathrm{T} 0))$, where Ns is the number $\log _{10}$ of specific bacteria in a specific test sample (i.e. treated with flavanol), $\mathrm{Nc}$ is the number $\log _{10}$ of specific bacteria in the control (no addition of flavanol), T1 is a specific time point and T0 is the $0 \mathrm{~h}$ time point. The control vessel contained only basal medium and faecal slurry $(1: 10, w / v)$ without any addition of flavanols.

\section{High-performance liquid chromatography analysis}

Analysis of flavanols and metabolites in the batch-culture vessels was assessed using a Hewlett-Packard 1100 series chromatograph (Hewlett-Packard, Palo Alto, CA, USA) equipped with a diode array detector and linked to the HP Chemstation Software system. Batch-culture supernatant fraction samples were diluted 1:5 in Milli-Q water before analysis using a reversed-phase C18 Nova-Pak column $(4.6 \times 250 \mathrm{~mm})$ (Waters Co.) with $4 \mu \mathrm{m}$ particle size. The mobile phase consisted of: (A) aqueous methanol $(5 \%)$ in 5 M-hydrochloric acid $(0.1 \%)$ and (B) acetonitrile-methanol (1:1) and was pumped through the column at $0.7 \mathrm{ml} / \mathrm{min}$. The following gradient system was used, where the percentage of solvent $\mathrm{B}$ was increased at the following times ( $\mathrm{min} / \% \mathrm{~B}$ ): 0/0, 5/5, 40/50, $60 / 5$ for detection of all compounds; the total run time was $60 \mathrm{~min}$. The eluant was monitored by photodiode array detection at $280 \mathrm{~nm}$ and spectra of products obtained over the 220 $600 \mathrm{~nm}$ range. Calibration curves of catechin and epicatechin were constructed using authentic standards $(0-100 \mu \mathrm{M})$ and in each case were found to be linear with correlation coefficients of $>0.995$.

Chromatographic chiral analyses were performed on HP 1100 Series HPLC (Hewlett-Packard) coupled to a fluorescence detector. Separations were carried out using a $4.6 \times 250 \mathrm{~mm}$; $5 \mu \mathrm{m}$ Sumichiral OA-7000 column (Sumika Chemical Analysis Service Ltd, Singapore) with a $10 \mu$ l injection volume. The binary mobile phase consisted of (A) $0.1 \%$ acetic acid in water and (B) $0.1 \%$ acetic acid in acetonitrile, with the isocratic separation carried out using $15 \%$ solvent $\mathrm{B}$. The flow rate of $0.250 \mathrm{ml} / \mathrm{min}$ was used throughout the run, which lasted $100 \mathrm{~min}$. Standards and samples were detected using a fluorescence detector with an excitation wavelength of $276 \mathrm{~nm}$ and emission wavelength of $316 \mathrm{~nm}$. Confirmation of the structure of (+)-epicatechin and (-)-epicatechin was confirmed by retention time, spectral mapping and by spiking with authentic standards.

\section{Measurement of antioxidant potential of batch-culture mixtures}

The FRAP assay was used as a direct measurement of the total antioxidant potential of each sample. The assay was performed as previously described ${ }^{(23)}$ with minor modifications. Briefly, sodium acetate trihydrate $(300 \mathrm{~mm}), 2,4,6$-tripyridyl-s-triazine $(10 \mathrm{~mm})$ in $\mathrm{HCl}(40 \mathrm{~mm})$ and $\mathrm{FeCl}_{3} \cdot 6 \mathrm{H}_{2} \mathrm{O}(20 \mathrm{~mm})$ were combined in a 10:1:1 ratio to obtain the FRAP reagent. Sample analysis was preformed on a ninety-six-well plate where $40 \mu \mathrm{l}$ of appropriately diluted sample were added to $300 \mu \mathrm{l}$ FRAP reagent in each well. Samples were analysed in triplicate. Plates were analysed using a GENios pro (Tecan, Theale, Berks, UK) spectrophotometer linked to the Magellan software program. The change of absorbance at $593 \mathrm{~nm}$ over $4 \mathrm{~min}$ is proportional to the combined (total antioxidant) FRAP value of the antioxidants in the sample. Untreated faecal supernatant fractions from the batch cultures were used as control and ascorbic acid was used as the positive control.

\section{Statistical analysis}

Each flavanol monomer was tested at two concentrations $(150 \mathrm{mg} / \mathrm{l}$ or $1000 \mathrm{mg} / \mathrm{l})$ in batch cultures inoculated with faecal samples collected from three individual donors. Experiments were performed in duplicate (i.e. each donor donated a faecal sample on two separate occasions). For bacterial enumeration work, changes in bacterial groups, calculated using the 'index of specific bacteria' (see Bacterial enumeration section), were expressed as mean values and standard deviations. A paired Student's $t$ test was used to test for significant differences at $10 \mathrm{~h}$ for flavanols $(150 \mathrm{ml} / \mathrm{l})$ and $17 \mathrm{~h}$ for flavanols $(1000 \mathrm{mg} / \mathrm{l})$ between the control vessel and flavanol-containing vessels. The rationale for the selection of these time points is discussed in the Results section. For the antioxidant potential work (FRAP assay) the results were expressed as mean values and standard deviations and significance was analysed using paired Student's $t$ tests. Significant differences in batch-culture flavanol levels were assessed using an unpaired $t$ test with Welsh's correction. 


\section{Results}

\section{Metabolism of flavanols by human faecal bacteria}

We applied a $\mathrm{pH}$-controlled, stirred, batch-culture fermentation system inoculated with a faecal slurry $(1: 10, \mathrm{w} / \mathrm{v})$ to determine the metabolism of $(-)$-epicatechin and $(+)$-catechin in a competitive microbial environment that mimics the distal large intestine. Fermentation suspensions were treated with $(-)$-epicatechin or $(+)$-catechin, and samples were collected as a function of time and analysed by HPLC for characterisation and quantification of intact flavanols and derived bacterial metabolites. Fig. 1 shows representative HPLC chromatograms and related spectral peak information for the metabolism of (-)-epicatechin by the microflora. (-)-Epicatechin was degraded progressively over a $24 \mathrm{~h}$ period but was not fully degraded in this time frame. Three major metabolites with similar spectral characteristics were observed at retention times of 18.4 (A1), 19.8 (A2) and 24.3 (A3) min, suggesting that they are more polar than epicatechin itself (Fig. 1 (A)). The metabolite at $18.4 \mathrm{~min}$ (A1) was identified as $5-\left(3^{\prime}, 4^{\prime}-\right.$ dihydroxyphenyl)- $\gamma$-valerolactone by MS, with analysis indicating that its amount increased as a function of exposure time (Fig. 1 (B)). Metabolite A2 was characterised by LCMS/MS to be phenylpropionic acid $\left(\mathrm{m} / \mathrm{z}, 151\right.$; $\left.\left[\mathrm{M}+\mathrm{H}^{+}\right]^{+}\right)$ whilst A3 was determined to be 5-phenyl- $\gamma$-valerolactone $\left(m / z, 191 ;\left[\mathrm{M}+\mathrm{H}^{+}\right]^{+}\right)$.

When $(+)$-catechin was incubated under the same conditions, the formation of four major, more polar, metabolites was observed at 11.3 (B2), 19.4 (B1), 24.0 (B3) and 25.6 (B4) min retention times (Fig. 2 (B)) over the course of $24 \mathrm{~h}$. The major metabolite formed at $10 \mathrm{~h}$ (B4) was determined to be either (+)-epicatechin or (-)-epicatechin by comparison with authentic standards and LC-MS/MS analysis (Fig. 2 (B)). Chiral separation of this sample by HPLC further identified this peak as (+)-epicatechin (Fig. 3). Metabolite B1, which also appeared after $10 \mathrm{~h}$ of incubation, eluted at a similar retention time to A1 and had similar spectral characteristics. Mass spectral analysis of B1 confirmed that this metabolite was also 5-( $3^{\prime}, 4^{\prime}$-dihydroxyphenyl)- $\gamma$-valerolactone (molecular weight of 207 detected in negative ion mode) and B3 was 5-phenyl- $\gamma$-valerolactone $\left(\mathrm{m} / z, 191 ;\left[\mathrm{M}+\mathrm{H}^{+}\right]^{+}\right)$. The rate of $(+)$-catechin metabolism by the microflora was significantly greater than that observed for $(-)$-epicatechin, with levels of $(+)$-catechin significantly lower at $4 \mathrm{~h}(P<0.01)$ and $8 \mathrm{~h}(P<0.001)$ at the $150 \mathrm{mg} / \mathrm{l}$ exposure (Fig. $4(\mathrm{~A}))$ and at $8 \mathrm{~h}(P<0 \cdot 01), 10 \mathrm{~h}$ and $17 \mathrm{~h}(P<0 \cdot 001)$ at the $1000 \mathrm{mg} / 1$ exposure (Fig. $4(\mathrm{~B}))$. This appeared to be due in part to the conversion of $(+)$-catechin to $(+)$-epicatechin during catechin fermentation experiments (Fig. 3). Degradation of both flavanols was influenced by individual variation in the composition of the faecal microflora, yielding relatively large inter-individual variation (Fig. 4). However, although the time course for different donors varied slightly, all donors yielded the same metabolites for $(-)$-epicatechin and $(+)$-catechin. No epimerisation of $(-)$-epicatechin or $(+)$-catechin was observed when flavanols were added to batch-culture systems without faecal inoculum (data not shown). In control experiments, no degradation of either $(+)$-catechin or ( - -epicatechin was observed when flavanols $(1000 \mathrm{mg} / \mathrm{l})$ were incubated in anaerobic batchculture vessels for $24 \mathrm{~h}$ without faecal inoculation.
Microflora-induced metabolism of both (-)-epicatechin and $(+)$-catechin was also observed in the presence of the favourable bacterial carbon sources, sucrose and the known prebiotic, FOS (Fig. 5), indicating bacterial metabolism of flavanols occurs even in the presence of preferential energy sources. Previous investigations have indicated that flavonoids may be metabolised by the gut microflora, although this is the first time their metabolism has been observed in the presence of alternate energy sources, a model which is more reflective of in vivo conditions. The extent of (-)-epicatechin metabolism by bacteria was significantly greater at both $8 \mathrm{~h}$ and $10 \mathrm{~h}(P<0.01)$ in the presence of both sucrose and FOS, with the combination of $(-)$-epicatechin $+1 \%$ FOS resulting in the greatest reduction in flavanol relative to $(-)$-epicatechin alone (Fig. 5 (A)). This was also apparent with (+)-catechin metabolism, with both sucrose and FOS significantly increasing the extent of bacterial $(+)$-catechin metabolism at $4 \mathrm{~h}$ $(P<0.01), 8 \mathrm{~h}$ and $10 \mathrm{~h}(P<0.001)$ (Fig. $5(\mathrm{~B}))$. There were no significant differences between the flavanol + sucrose treatments and flavanol + FOS treatments $(P>0 \cdot 05)$.

\section{Changes in antioxidant potential}

The metabolism of both flavanols over the $24 \mathrm{~h}$ batch-culture incubation period was paralleled by decreases in the total antioxidant capacity of the batch-culture mixtures. Batch cultures containing (-)-epicatechin $(150 \mathrm{mg} / \mathrm{l})$ showed a time-dependent reduction in antioxidant capacity, which achieved significance at 17 and $24 \mathrm{~h}$ of incubation with the microflora. Similarly, there was a decrease in antioxidant activity in batch cultures exposed to (+)-catechin (Fig. 6). However, vessels treated with either $(+)$-catechin or $(-)$-epicatechin had increased antioxidant activity compared with untreated vessels at all time points with the exception of $24 \mathrm{~h}$ (Fig. 6).

\section{Flavanol-induced changes in specific bacterial groups}

In order to assess the changes in bacterial populations in response to $(-)$-epicatechin or $(+)$-catechin exposure, we utilised FISH analysis. Previous in vitro and in vivo studies designed to assess potential prebiotic effects of different substrates have successfully utilised FISH analysis to enumerate different bacterial groups found in the human faecal microflora and their changes over time ${ }^{(24,25)}$. FISH analysis was performed at time points where more than $50 \%$ of flavanol had been metabolised by the faecal microflora in the mixed batch culture. This was revealed by HPLC analysis to be $10 \mathrm{~h}$ for experiments with $(+)$-catechin and $(-)$-epicatechin at $150 \mathrm{mg} / \mathrm{l}$ and $17 \mathrm{~h}$ at $1000 \mathrm{mg} / \mathrm{l}$ (Fig. 4). (-)-Epicatechin $(150 \mathrm{mg} / \mathrm{l})$ caused a significant increase in the growth of the beneficial bacterial group, Eubacterium rectale-C. coccoides (Fig. 7 (A)), as revealed by an increase in the number of this bacterial group in vessels containing (-)-epicatechin, compared with the number found in control vessels, at the same time point (index of specific bacteria). However, (-)epicatechin did not induce any other significant changes in the microflora either at the 150 or $1000 \mathrm{mg} / \mathrm{l}$ concentration. The significant increase in Eubacterium rectale-C. coccoides was only observed at the lower concentration of (-)-epicatechin (Fig. 7 (A)). There was no significant difference between 

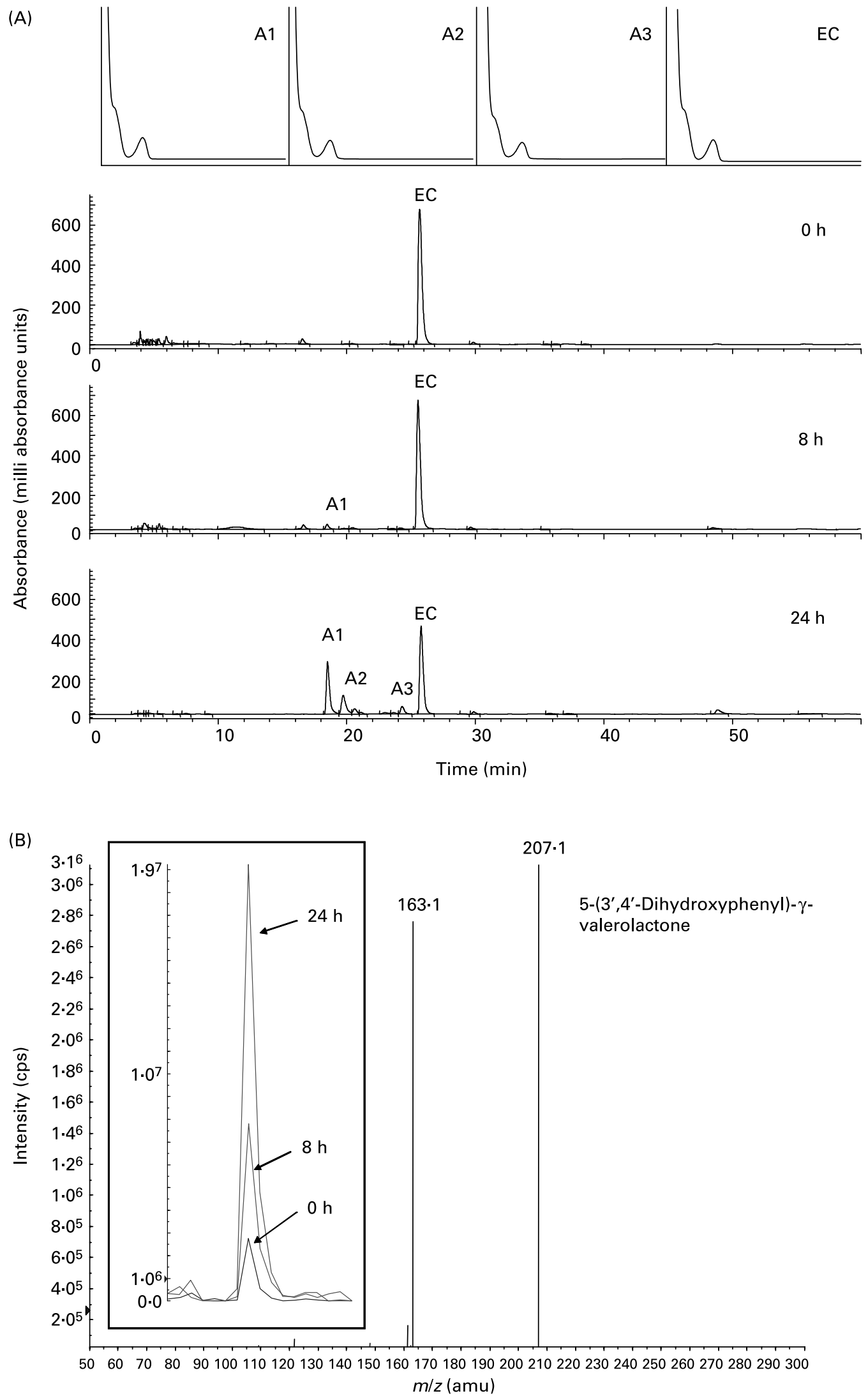

Fig. 1. Bacterial-dependent metabolism of (-)-epicatechin (EC). (A) Typical HPLC chromatogram with photodiode array spectra (200-600 nm) of faecal supernatant fraction derived from the batch culture with $\mathrm{EC}$ at $1000 \mathrm{mg} / \mathrm{l}$ concentration after 0,8 and $24 \mathrm{~h}$ of incubation with human colonic bacteria. EC and three new compounds (A1-A3) were detected. (B) LC-MS/MS analysis of peak A1 confirming the presence of 5 - $\left(3^{\prime}, 4^{\prime} \text {-dihydroxyphenyl)- } \gamma \text {-valerolactone [n- } \mathrm{H}^{+}\right]^{+} \mathrm{m} / \mathrm{z} 207$.

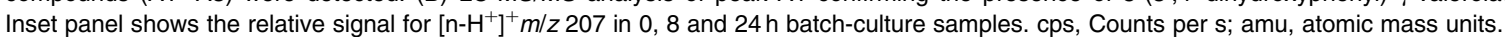



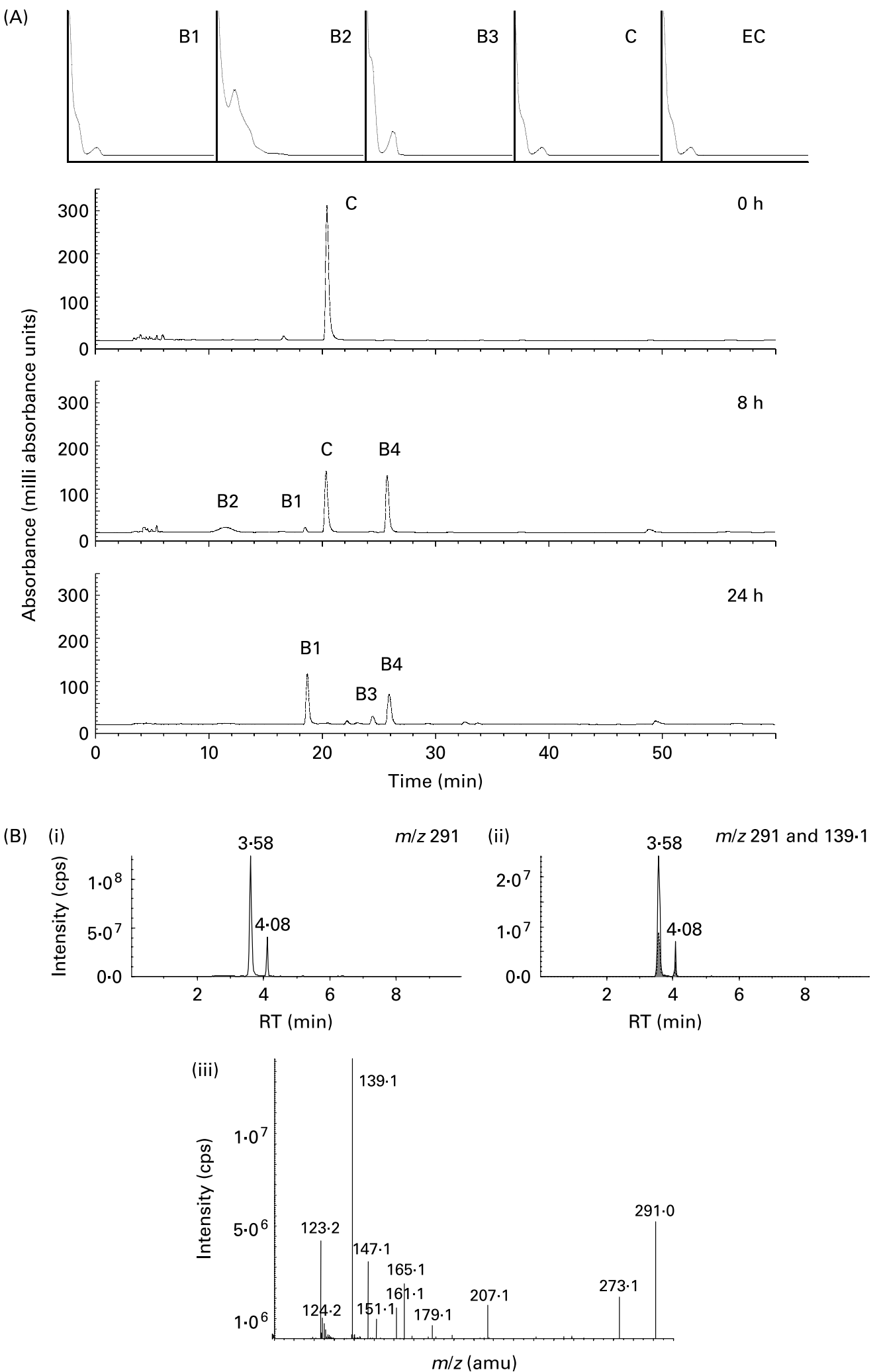

Fig. 2. Bacterial-dependent metabolism of (+)-catechin. (A) Typical HPLC chromatogram with photodiode array spectra (200-600 nm) of faecal supernatant fraction derived from the batch culture with $(+)$-catechin at $1000 \mathrm{mg} / \mathrm{l}$ concentration after 0,8 and $24 \mathrm{~h}$ of incubation with human colonic bacteria. $(+)$-Catechin $(C)$ and four new compounds (B1-B4) were detected. (B) LC-MS/MS analysis of the same sample indicating the presence of a compound with $m / z 291$ at two retention times. (i) Selected ion scan of $\left[n-H^{+}\right]^{+} m / z 291$; (ii) selected ion scan $\left[n-H^{+}\right]^{+} m / z 291$ overlaid with $\left[n-H^{+}\right]^{+} m / z 139$ (घ); (iii) fragment ion spectrum of epicatechin showing the abundant signal at $\mathrm{m} / \mathrm{z} 139$ representing the retro Diels-Alder product deriving from the A-ring. cps, Counts per s; RT, retention time; amu, atomic mass units. 
(A)

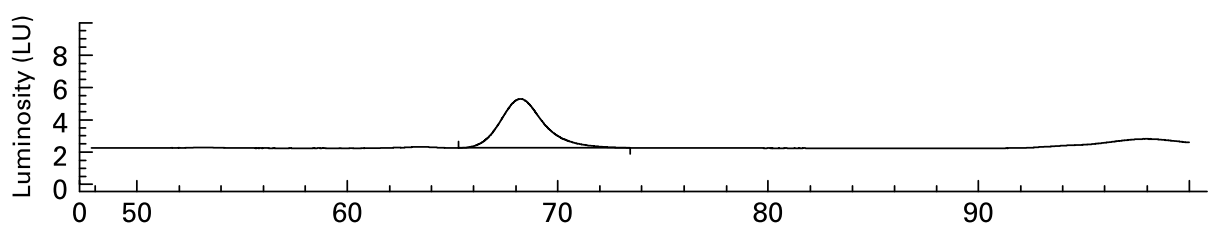

(B)

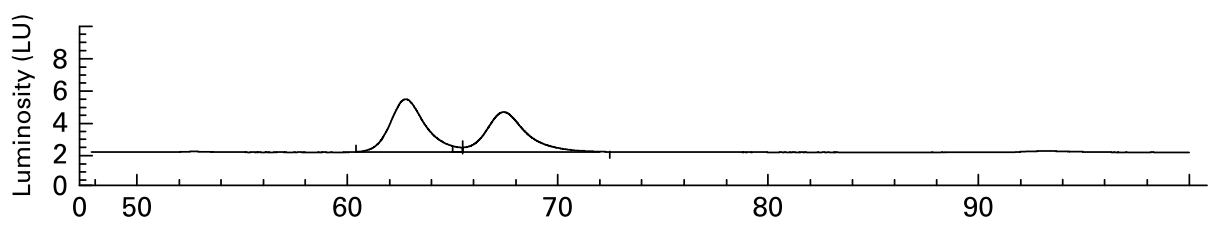

(C)

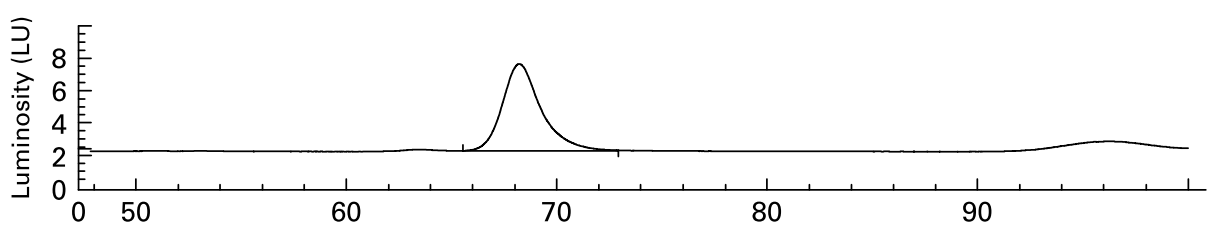

(D)

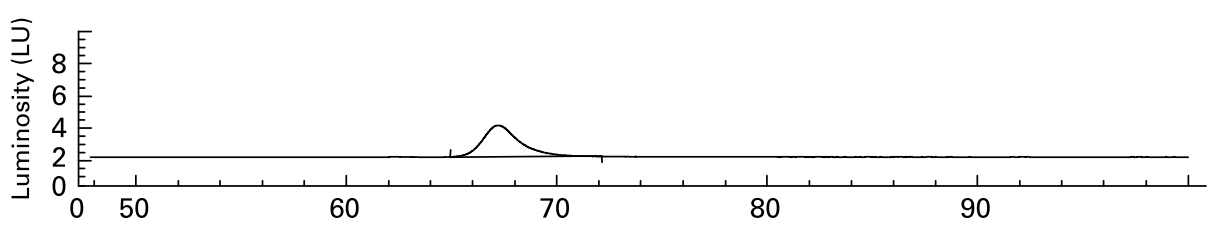

(E)

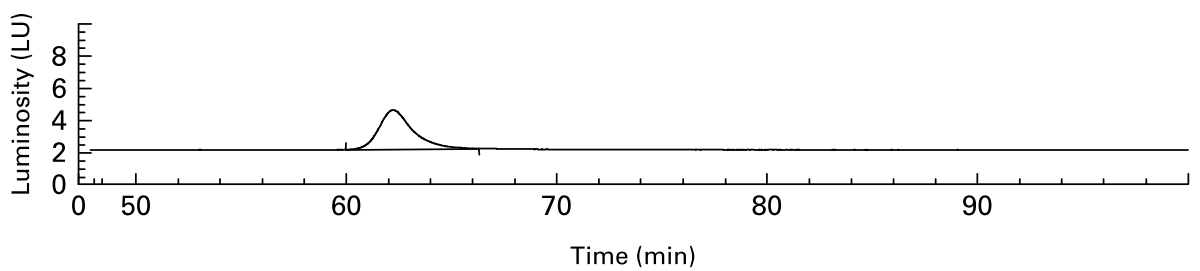

Fig. 3. Chiral separation of peak B4 derived from the batch culture with (+)-catechin following $10 \mathrm{~h}$ fermentation. Indication that metabolite B4 (see Fig. 2) relates to $(+)$-epicatechin. Detection was by fluorescence (excitation $276 \mathrm{~nm}$ and emission $316 \mathrm{~nm}$ ). The sample containing peak B4 (A) was spiked with authentic $(-)$ epicatechin (B) or (+)-epicatechin (C). (D) (+)-Epicatechin standard; (E) (-)-epicatechin standard. LU, luminosity units.

the low flavanol $(150 \mathrm{mg} / \mathrm{l})$ treatment and the high flavanol $(1000 \mathrm{mg} / \mathrm{l})$ treatment $(P>0.05)$.

In contrast, $(+)$-catechin exposure resulted in a greater modification in the growth of the bacterial groups compared with that seen with (-)-epicatechin (Fig. 7 (B)). There was a significant decrease in the growth of the $C$. histolyticum group at the $1000 \mathrm{mg} / \mathrm{l}$ concentration, relative to control, and a marked increase in the growth of the beneficial bacterial group of $C$. coccoides-Eubacterium rectale at both the $150 \mathrm{mg} / \mathrm{l}$ and $1000 \mathrm{mg} / \mathrm{l}$ exposure concentrations. Furthermore, there were increases in both Lactobacillus spp. and Bifidobacterium spp. following $(+)$-catechin $(150 \mathrm{mg} / \mathrm{l}$ exposure $)$, which achieved significance for the latter. There were also small, but significant increases, in the growth of E. coli in response to catechin $(150 \mathrm{mg} / \mathrm{l})$ incubation with the bacterial culture. There was no significant difference in the total number of microbes measured in vessels containing either flavanol (150 or $1000 \mathrm{mg} / \mathrm{l})$, compared with the total number measured in the control vessels.

\section{Discussion}

In recent years there has been much interest in the development of nutritional products that are based on a prebiotic principle. Prebiotics are non-digestible food ingredients that beneficially affect the host by selectively stimulating the growth and/or activity of one, or a limited number of, beneficial bacteria in the colon ${ }^{(8)}$. Although the effects of many carbohydrates have been investigated as potential prebiotics, for example inulin and FOS, few studies have considered the prebiotic potential of other dietary components. Flavonoids are potential prebiotic candidates as they are known to undergo limited absorption and metabolism in the upper gastrointestinal tract (stomach, duodenum and jejunum), with the majority reaching the large intestine intact ${ }^{(26)}$ where approximately $200 \mathrm{~g}$ of material (water, bacteria, food particles and mucus) are present at any given time ${ }^{(27)}$. Green tea contains up to $8.5 \mathrm{mg}(-)$-epicatechin $/ 100 \mathrm{ml}$ and $2.8 \mathrm{mg}$ 
$(+)$-catechin $/ 100 \mathrm{ml}^{(28)}, 100 \mathrm{~g}$ dark chocolate contains, on average, $42 \mathrm{mg}(-)$-epicatechin and $12 \mathrm{mg}(+)$-catechin. In the present study we show that the metabolism of the flavanol monomers, (-)-epicatechin and (+)-catechin, by a human bacterial population representative of the distal part of the human large intestine leads to changes in the growth of specific bacterial populations. Specifically, $(+)$-catechin, and to a lesser extent $(-)$-epicatechin, had a positive effect on the growth of $C$. coccoides-Eubacterium rectale, a bacterial group which has been associated with beneficial effects at the cellular and systemic level due to their ability to generate SCFA by saccharolytic metabolism ${ }^{(29)}$. The flavanol-induced increases in this bacterial group seen in our investigations may result in an increased potential for saccharolytic

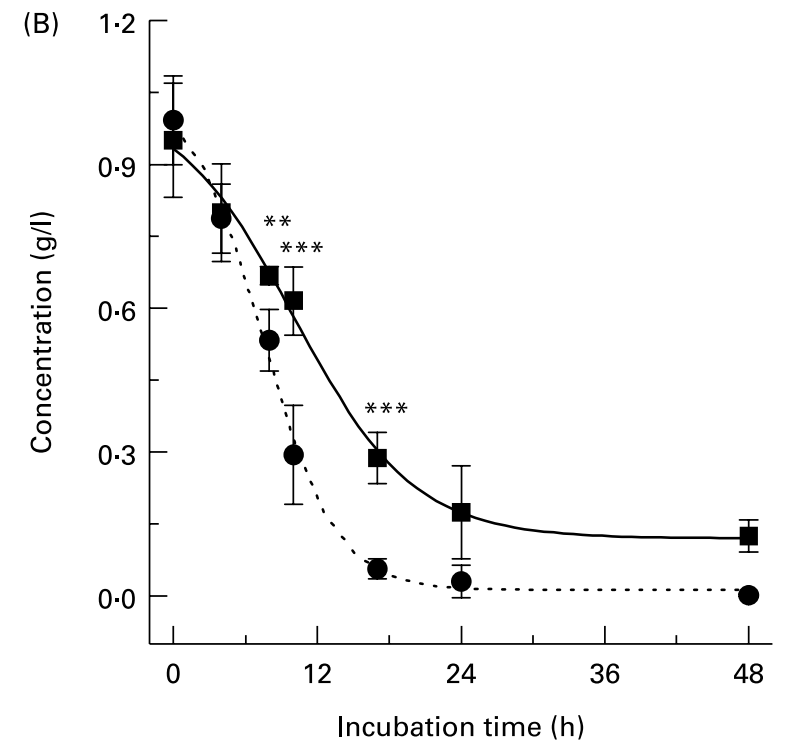

Fig. 4. Metabolism profiles of $(+)$-catechin $(\bullet)$ and $(-)$-epicatechin $(\square)$ from three individual donors performed in duplicate. (A) Flavanol (150 mg/l); (B) flavanol $(1000 \mathrm{mg} / \mathrm{l})$. Results are expressed as amount $(\mathrm{g} / \mathrm{l})$ in batch-culture medium. Values are means $(n 6)$, with standard deviations represented by vertical bars. Significant difference between $(+)$-catechin and (-)-epicatechin concentrations in batch-culture vessels: ${ }^{\star \star} P<0.01$, ${ }^{\star \star \star} P<0.001$ metabolism in the large intestine, an event believed to be beneficial primarily due to the production of the SCFA acetate, propionate and butyrate. The generation of such SCFA in the large intestine is considered positive for gut health as they have been linked to an inhibition of pre-neoplastic proliferation and an acceleration of the conversion of cholesterol into bile acids ${ }^{(30)}$.

$(+)$-Catechin exposure also resulted in the small, but significant, increase in the growth of bifidobacteria, a group which has been strongly associated with positive effects in the large intestine ${ }^{(8)}$, through their ability to inhibit the
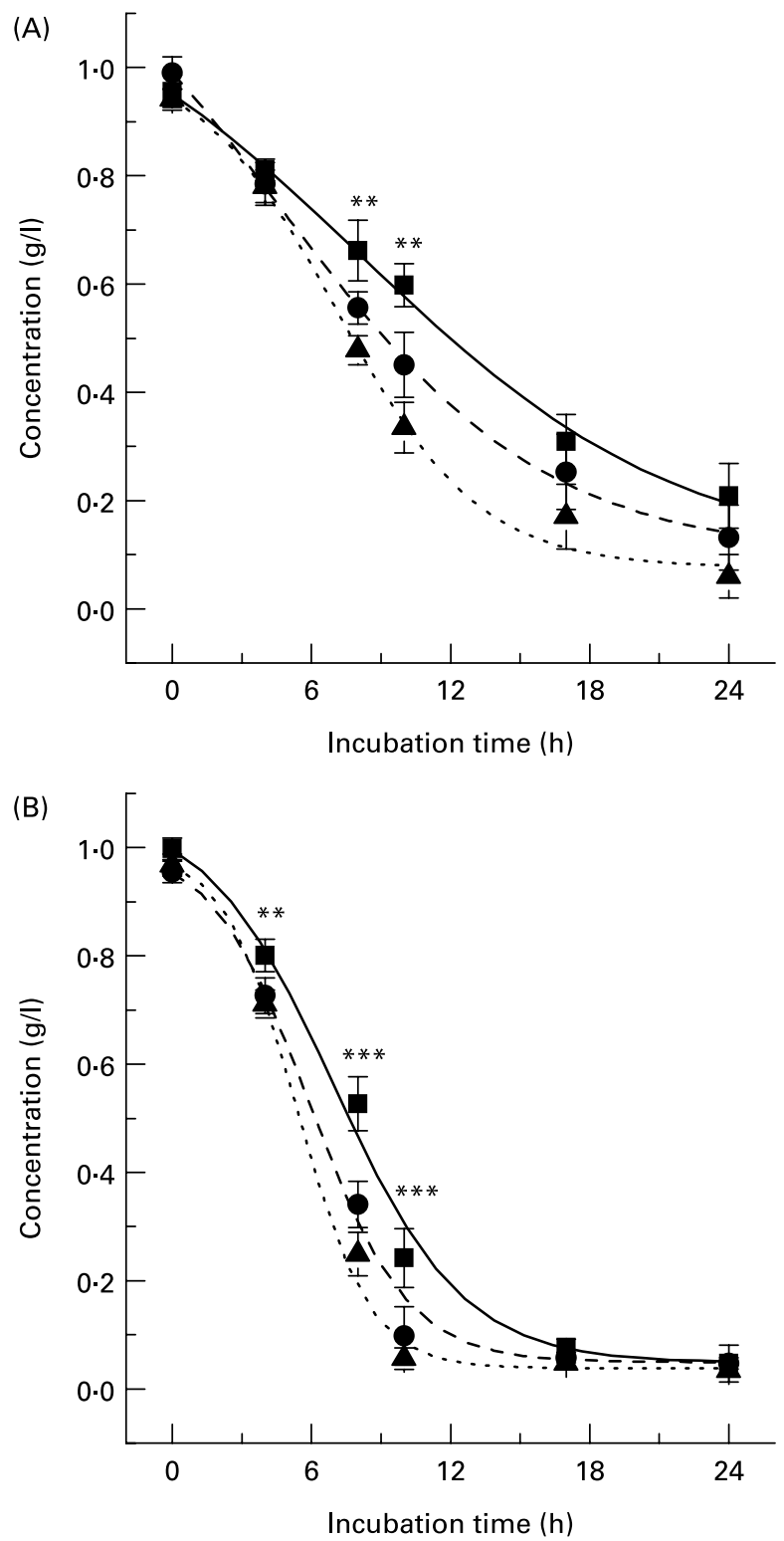

Fig. 5. Flavanol metabolism in the presence of carbohydrates. (-)-Epicatechin $(\mathrm{A})$ and $(+)$-catechin $(\mathrm{B})$ assimilation profiles in $\mathrm{pH}$-controlled batch cultures in the presence of $1 \%$ fructo-oligosacchrides (FOS) or $1 \%$ sucrose. $(\mathrm{A})$ : $(\boldsymbol{\square}),(-)$-Epicatechin; $(\bullet),(-)$-epicatechin $+1 \%$ sucrose; $(\boldsymbol{\Lambda}),(-)$-epicatechin $+1 \%$ FOS. $(\mathrm{B})$ : $(\boldsymbol{\square}),(+)$-Catechin; $(\bullet),(+)$-catechin $+1 \%$ sucrose; $(\boldsymbol{\Lambda}),(+)$-catechin $+1 \%$ FOS. Results are expressed as concentration $(\mathrm{g} / \mathrm{l})$ in batch-culture medium. Values are means $(n$ 6), with standard deviations represented by vertical bars. Mean values of flavanol only and flavanol + FOS/sucrose were significantly different: ${ }^{\star \star} P<0.01,{ }^{\star \star \star} P<0.001$. 
growth of pathogenic micro-organisms. $(+)$-Catechin-induced changes in bifidobacteria may stimulate the generation of organic acids, such as acetic and lactic acid in the colon ${ }^{(21)}$ and may inhibit the ability of potential pathogenic species to colonise the gut epithelial lair ${ }^{(15)}$. Furthermore, (+)-catechin-induced decreases in the $C$. histolyticum group, a group of mostly proteolytic bacteria, may be particularly relevant in the distal large intestine where proteolytic metabolism has been proposed to contribute to the progression of colonic cancer and the onset of inflammatory bowel disease ${ }^{(31)}$. Bacterial groups, such as Bacteroides and Clostridium favour proteolytic fermentation, which results in the production of ammonia, thiols, amines and indoles, endproducts that have been connected with neoplastic growth and cancer ${ }^{(32,33)}$. It is more difficult to interpret the small $E$. coli increases observed in response to $(+)$-catechin exposure, as the probe used in our investigations enumerates both commensal and pathogenic strains of E. coli. Further investigation is required to identify the specific strains affected in our system. However $E$. coli is one of the most common bacteria inhabiting the intestinal tract of humans, and thus the observed increase is unlikely to represent a change in the small number of strains responsible for diarrhoeal diseases ${ }^{(34)}$.

The significant changes in bacterial composition in response to $(+)$-catechin were accompanied by its rapid conversion to $(+)$-epicatechin. Recently, Lee et al. reported similar conversions of catechin to epicatechin upon bacterial incubation, although in their study they did not confirm the specific isomeric form as was done in the present investigation ${ }^{(22)}$. In contrast to the present study, we only observed the conversion of $(+)$-catechin to $(+)$-epicatechin in the presence of bacteria, suggesting that it is the microflora that mediate this conversion. Moreover,

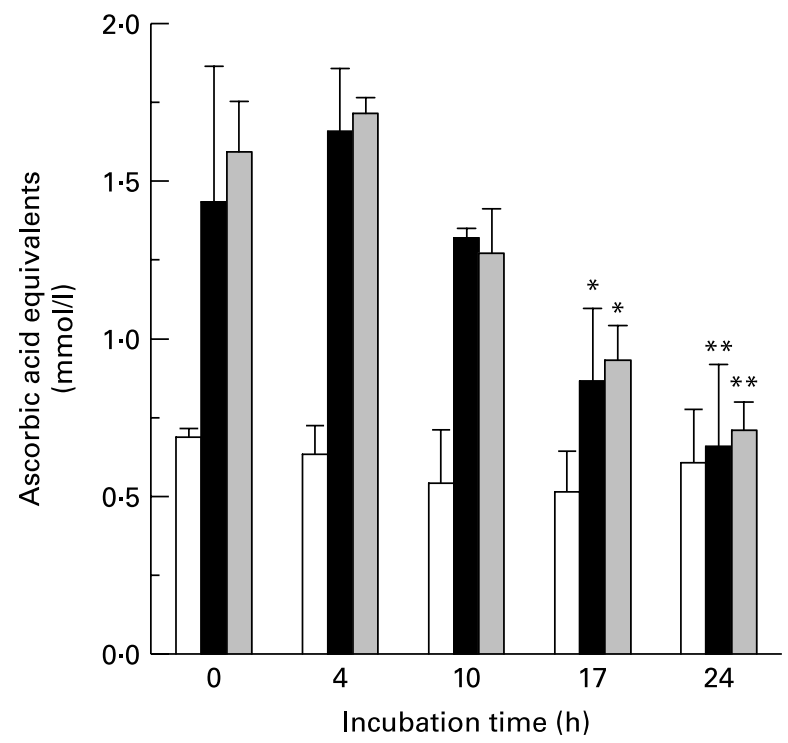

Fig. 6. The effect of bacterial metabolism on antioxidant potential. Changes in the antioxidant potential of batch-culture mixtures over time following incubation with basal medium only $(\square),(-)$-epicatechin $(150 \mathrm{mg} / \mathrm{l})(\boldsymbol{\square})$ and catechin $(150 \mathrm{mg} / \mathrm{l})(\square)$ were determined by the ferric-reducing antioxidant power assay. The antioxidant activity is expressed as ascorbic acid equivalents $(\mu \mathrm{mol} / \mathrm{l})$. Values are means $(n 6)$, with standard deviations represented by vertical bars. Mean antioxidant capacity was significantly decreased compared with that at time $0:{ }^{\star} P<0.05,{ }^{\star \star} P<0.01$. our data indicate that the reduction in flavanol monomer concentration in batch-culture vessels is entirely due to specific bacterial-dependent metabolism, rather than unspecific bacterial-independent degradation, as we did not observe degradation of either flavanol, for example by their oxidation, in our anaerobic batch-culture environment. As there were no major differences in the other metabolites produced from either $(+)$ catechin and (-)-epicatechin, with both yielding 5-(3',4'-dihydroxyphenyl)- $\gamma$-valerolactone, 5-phenyl- $\gamma$-valerolactone and phenylpropionic acid following incubation with the microflora, the greater influence of $(+)$-catechin on the growth of specific microflora may be the result of the bacterial-driven formation of $(+)$-epicatechin from $(+)$-catechin.

The formation of valerolactone species from flavanols in the large intestine has previously been reported ${ }^{(20,21,35,36)}$ and such metabolic products may represent potential bioactive metabolites of flavanols in vivo following their absorption into the circulation via the gut wall. This generation of metabolites
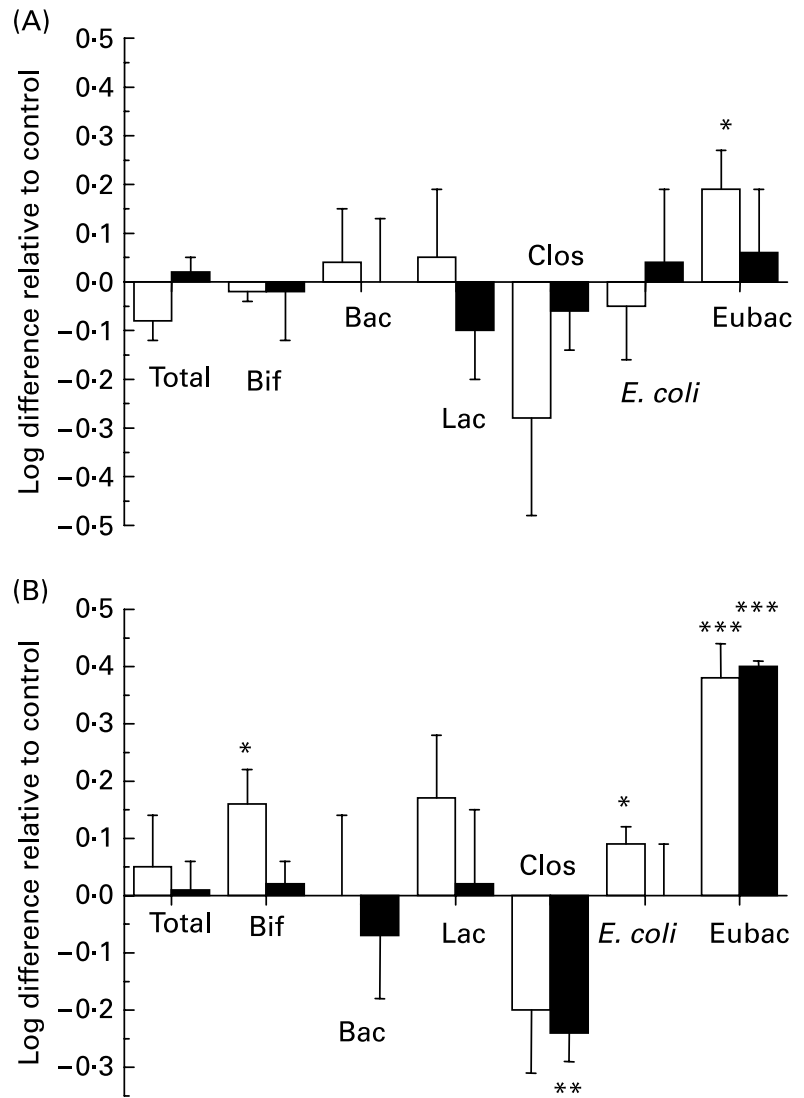

Fig. 7. Influence of flavanol monomers on the colonic microflora. Changes in the bacterial populations during the fermentation of $(-)$-epicatechin $(A)$ and $(+)$-catechin $(B)$ in a $\mathrm{pH}$-controlled faecal batch culture. Samples were taken at 10 and $17 \mathrm{~h}$ for $150 \mathrm{mg} / \mathrm{l}(\square)$ and $1000 \mathrm{mg} / \mathrm{l}(\square)$ concentrations, respectively and analysed by fluorescent in situ hybridisation. Bif, Bifidobacterium spp.; Bac, Bacteroides spp.; Lac, Lactobacillus/Enterococcus spp.; Clos, Clostridium histolyticum group; E. coli, Escherichia coli; Eubac, C. coccoides-Eubacterium rectale group. Bacterial changes, calculated using the 'index of specific bacteria', are expressed as $\log _{10}$ cells $/ \mathrm{ml}$. Changes in bacterial growth were calculated by comparing the number of a specific bacterial group in the treatment group with the number found in a control group, at the same time point. Values are means $(n 6)$, with standard deviations represented by vertical bars. Mean value was significantly different from that of the control: ${ }^{*} P<0.05,{ }^{\star \star *} P<0.001$. 
was also marked by a general reduction in the antioxidant potential of the batch-culture mixture, suggesting that species such as valerolactones possess lower antioxidant potential compared with native flavanols. However, despite this significant reduction in antioxidant activity, regular flavanol-rich food consumption is likely to result in a general increase in the antioxidant capacity in the large intestine. This may be relevant in reducing oxidative stress in the large intestine that occurs as a result of inflammatory events experienced during inflammatory bowel disease ${ }^{(37)}$. Previous studies have shown that dietaryderived antioxidants may exert a beneficial effect on large-intestinal disorders, such as inflammatory bowel diseases, where they act to reduce the levels of certain cytokines and scavenge oxidation products derived from other dietary factors or from bacterial oxidative metabolism ${ }^{(38,39)}$.

The generation of metabolic products following incubation with the bacteria suggests that flavanols may be potential nutrient sources for the bacteria. Indeed, both flavanols were metabolised by the human colonic bacteria even in the presence of carbohydrate energy sources, notably sucrose and the pre-biotic FOS, suggesting that the metabolism of flavanols may occur even in the presence of more favourable carbon sources. These observations suggest that these flavanol monomers may be capable of influencing the large-intestinal bacterial population even in the presence of other nutrients, such as carbohydrates and proteins. Our data indicate that flavanols have an ability to influence the growth of specific large-intestinal bacteria and that this ability may be linked to specific metabolic transformations, such as the bacterial conversion of $(+)$-catechin to $(+)$-epicatechin. However, we acknowledge that this is an in vitro investigation using a batch-culture model and it may not necessarily explain with certainty the effects of flavanols in humans. Future human intervention studies will provide further insight into the potential of flavanol monomers to act as prebiotics in the human large intestine in vivo.

\section{Acknowledgements}

The present study was supported by Mars Incorporated and a Reading University Endowment Trust Fund Studentship.

\section{References}

1. Graf BA, Milbury PE \& Blumberg JB (2005) Flavonols, flavones, flavanones, and human health: epidemiological evidence. J Med Food 8, 281-290.

2. Arts ICW \& Hollman PCH (2005) Polyphenols and disease risk in epidemiologic studies. Am J Clin Nutr 81, Suppl. 1, S317-S325.

3. Fisher NDL, Hughes M, Gerhard-Herman M \& Hollenberg NK (2003) Flavanol-rich cocoa induces nitric-oxide-dependent vasodilation in healthy humans. J Hypertens 21, 2281-2286.

4. Heiss C, Dejam A, Kleinbongard P, Schewe T, Sies H \& Kelm M (2003) Vascular effects of cocoa rich in flavan-3-ols. $J$ Am Med Assoc 290, 1030-1031.

5. Taubert D, Berkels R, Roesen R \& Klaus W (2003) Chocolate and blood pressure in elderly individuals with isolated systolic hypertension. J Am Med Assoc 290, 1029-1030.

6. Kuhnle G, Spencer JPE, Schroeter H, Shenoy B, Debnam ES, Srai SK, Rice-Evans C \& Hahn U (2000) Epicatechin and catechin are $O$-methylated and glucuronidated in the small intestine. Biochem Biophys Res Commun 277, 507-512.

7. Spencer JPE (2003) Metabolism of tea flavonoids in the gastrointestinal tract. J Nutr 133, S3255-S3261.

8. Gibson GR, Beatty ER, Wang X \& Cummings JH (1995) Selective stimulation of bifidobacteria in the human colon by oligofructose and inulin. Gastroenterology 108, 975-982.

9. Onoue M, Kado S, Sakaitani Y, Uchida K \& Morotomi M (1997) Specific species of intestinal bacteria influence the induction of aberrant crypt foci by 1,2-dimethylhydrazine in rats. Cancer Lett 113, 179-186.

10. Rastall RA (2004) Bacteria in the gut: friends and foes and how to alter the balance. J Nutr 134, S2022-S2026.

11. O'Mahony L, McCarthy J, Kelly P, et al. (2005) Lactobacillus and bifidobacterium in irritable bowel syndrome: symptom responses and relationship to cytokine profiles. Gastroenterology 128, $541-551$.

12. Saggioro A (2004) Probiotics in the treatment of irritable bowel syndrome. J Clin Gastroenterol 38, Suppl. 6, S104-S106.

13. Marteau PR (2002) Probiotics in clinical conditions. Clin Rev Allergy Immun 22, 255-273.

14. Gibson GR, Probert HM, Van Loo J, Rastall RA \& Roberfroid MB (2004) Dietary modulation of the human colonic microbiota: updating the concept of prebiotics. Nutr Res Rev 17, 259-275.

15. Gibson GR \& Fuller R (2000) Aspects of in vitro and in vivo research approaches directed toward identifying probiotics and prebiotics for human use. J Nutr 130, S391-S395.

16. Kleessen B, Hartmann L \& Blaut M (2001) Oligofructose and long-chain inulin: influence on the gut microbial ecology of rats associated with a human faecal flora. Br J Nutr 86, 291-300.

17. Wijnands MVW, Appel MJ, Hollanders VMH \& Woutersen RA (1999) A comparison of the effect of dietary cellulose and fermentable galacto-oligosaccharides, in a rat model of colorectal carcinogenesis: fermentable fibre confers greater protection than non-fermetable fibre in both high and low fat backgrounds. Carcinogenesis 20, 651-656.

18. Gibson GR, Beatty ER, Wang X \& Cummings JH (1995) Selective stimulation of Bifidobacteria in the human colon by oligofructose and inulin. Gastroenterology 108, 975-982.

19. Clavel T, Fallani M, Lepage P, et al. (2005) Isoflavones and functional foods alter the dominant intestinal microbiota in postmenopausal women. J Nutr 135, 2786-2792.

20. Deprez S, Brezillon C, Rabot S, Philippe C, Mila C, Lapierre C $\&$ Scalbert A (2000) Polymeric proanthocyanidins are catabolized by human colonic microflora into low-molecular-weight phenolic acids. $J$ Nutr 130, 2733-2738.

21. Li C, Lee MJ, Sheng S, et al. (2000) Structural identification of two metabolites of catechins and their kinetics in human urine and blood after tea ingestion. Chem Res Toxicol 13, 177-184.

22. Lee HC, Jenner AM, Low CS \& Lee YK (2006) Effect of tea phenolics and their aromatic fecal bacterial metabolites on intestinal microbiota. Res Microbiol 157, 876-884.

23. Benzie IFF \& Strain JJ (1996) The ferric reducing ability of plasma (FRAP) as a measure of "antioxidant power". The FRAP assay. Anal Biochem 239, 70-76.

24. Olano-Martin E, Gibson GR \& Rastall RA (2002) Comparison of the in vitro bifidogenic properties of pectins and pectic-oligosaccharides. J Appl Microb 93, 505-511.

25. Tuohy MK, Kolida S, Lustenberger MA \& Gibson RG (2001) The prebiotic effect of biscuits containing partially hydrolysed guar gum and fructo-oligosaccharides - a human volunteer study. Br J Nutr 86, 341-348.

26. Spencer JPE, Schroeter H, Rechner AR \& Rice-Evans C (2001) Bioavailability of flavan-3-ols and procyanidins: gastrointestinal tract influences and their relevance to bioactive forms in vivo. Antioxid Redox Signal 3, 1023-1039. 
27. Frayn KN (2003) Digestion and intestinal absorption. In Metabolic Regulation. A Human Perspective, 2nd ed. pp. 59-82 [KN Frayn, editor]. Oxford, UK: Blackwell Publishing.

28. Manach C, Scalbert A, Morand C, Remesy C \& Jimenez L (2004) Polyphenols: food sources and bioavailability. Am J Clin Nutr 79, 727-747.

29. Gibson RG (1999) Dietary modulation of the human gut microflora using the prebiotics oligofructose and inulin. J Nutr 129, 1438-1441.

30. Wilson M (2005) Short-chain fatty acids. In Microbial Inhabitants of Humans, pp. 388-390 [M Wilson, editor]. Cambridge, UK: Cambridge University Press.

31. Hughes R, Magee EAM \& Bingham S (2000) Protein degradation in the large intestine: relevance to colorectal cancer. Curr Issues Intest Microbiol 1, 51-58.

32. Matsui T, Matsukawa Y, Sakai T, Nakamura K, Aoike A \& Kawai K (1995) Effect of ammonia on cell-cycle progression of human gastric-cancer cells. Eur J Gastroenterol Hepatol 7, S79-S81.

33. Gibson SAW, McFarlan C, Hay S \& Macfarlane GT (1989) Significance of microflora in proteolysis in the colon. Appl Environ Microb 55, 679-683.
34. Stenutz R, Weintraub A \& Widmalm G (2006) The structures of Escherichia coli $O$-polysaccharide antigens. FEMS Microbiol Rev 30, 382-403.

35. Li C, Meng X, Winnik B, Lee MJ, Lu H, Sheng S, Buckley B \& Yang CS (2001) Analysis of urinary metabolites of tea catechins by liquid chromatography/electrospray ionization mass spectrometry. Chem Res Toxicol 14, 702-707.

36. Meselhy MR, Nakamura N \& Hattori M (1997) Biotransformation of (-)-epicatecbin 3-O-gallate by human intestinal bacteria. Chem Pharm Bull (Tokyo) 45, 888-893.

37. Garsetti M, Pellegrini N, Baggio C \& Brighenti F (2000) Antioxidant activity in human faeces. Br J Nutr 84, 705-710.

38. Pearson DC, Jourd'heuil D \& Meddings JB (1996) The antioxidant properties of 5-aminosalicylic acid. Free Radic Biol Med 21, 367-373.

39. Reimund JM, Allison AC, Muller CD, Dumont S, Kenney JS, Baumann R, Duclos B \& Poindron P (1998) Antioxidants inhibit the in vitro production of inflammatory cytokines in Crohn's disease and ulcerative colitis. Eur J Clin Invest 28, $145-150$. 